\title{
La contribution de l'analyse des espaces et du territoire des ateliers de réparation ferroviaire d'Arles à la connaissance du patrimoine industriel et urbain
}

The contribution of the analysis of the spaces and territories of the rail repair workshops of Arles to the knowledge of the industrial and urban heritage

\section{Coralie Grégoire}

\section{OpenEdition}

\section{Journals}

Édition électronique

URL : https://journals.openedition.org/rhcf/1828

DOI : 10.4000/rhcf.1828

\section{Éditeur}

Rails \& histoire

Édition imprimée

Date de publication : 1 décembre 2003

Pagination : $549-560$

ISBN : 0996-9403

ISSN : 0996-9403

\section{Référence électronique}

Coralie Grégoire, "La contribution de l'analyse des espaces et du territoire des ateliers de réparation ferroviaire d'Arles à la connaissance du patrimoine industriel et urbain », Revue d'histoire des chemins de fer [En ligne], 28-29 | 2003, mis en ligne le 06 janvier 2015, consulté le 22 avril 2022. URL : http:// journals.openedition.org/rhcf/1828; DOI : https://doi.org/10.4000/rhcf.1828

Ce document a été généré automatiquement le 22 avril 2022

Tous droits réservés 


\section{La contribution de l'analyse des espaces et du territoire des ateliers de réparation ferroviaire d'Arles à la connaissance du patrimoine industriel et urbain}

The contribution of the analysis of the spaces and territories of the rail repair workshops of Arles to the knowledge of the industrial and urban heritage

\section{Coralie Grégoire}

Construits de 1845 à 1856 et mis en service dès 1848, les Ateliers d'Arles doivent pourvoir aux besoins de la ligne Avignon-Marseille, ligne incorporée en 1857 à la Compagnie du Paris-Lyon-Méditerranée (PLM). Après 136 ans d'activité de construction et de réparation ferroviaires, ils ferment leurs portes le 31 décembre 1984. Reste aujourd'hui une friche industrielle d'environ onze hectares dont une partie est toujours utilisée par le service Fret de la SNCF pour de l'entreposage (fig. 1 et 2). On peut y voir des bâtiments de type industriel (vastes halles fermées, grands volumes; le plus vaste, la chaudronnerie, a une surface de $6000 \mathrm{~m}^{2}$ ) côtoyer d'autres édifices de facture plus classique (constructions à usage administratif, logements pour le personnel dirigeant). Le site et les bâtiments qui le composent sont les artefacts de l'histoire des Ateliers d'Arles et la manifestation physique d'un siècle et demi d'activité industrielle ferroviaire... 
Figure 1. Plan d'Arles : situation des Ateliers.

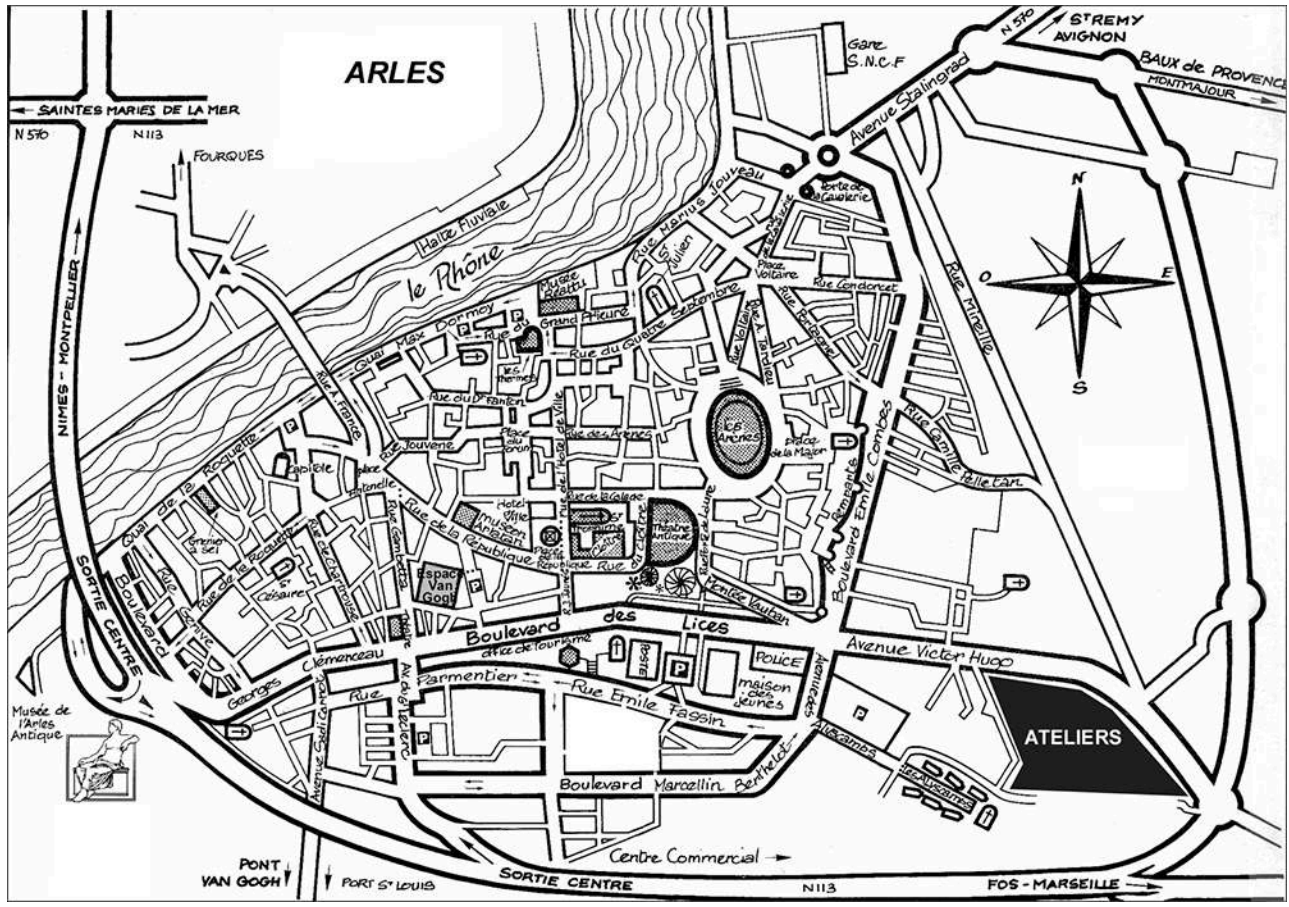

Figure 2. Les Ateliers dans la ville d'Arles, état actuel.

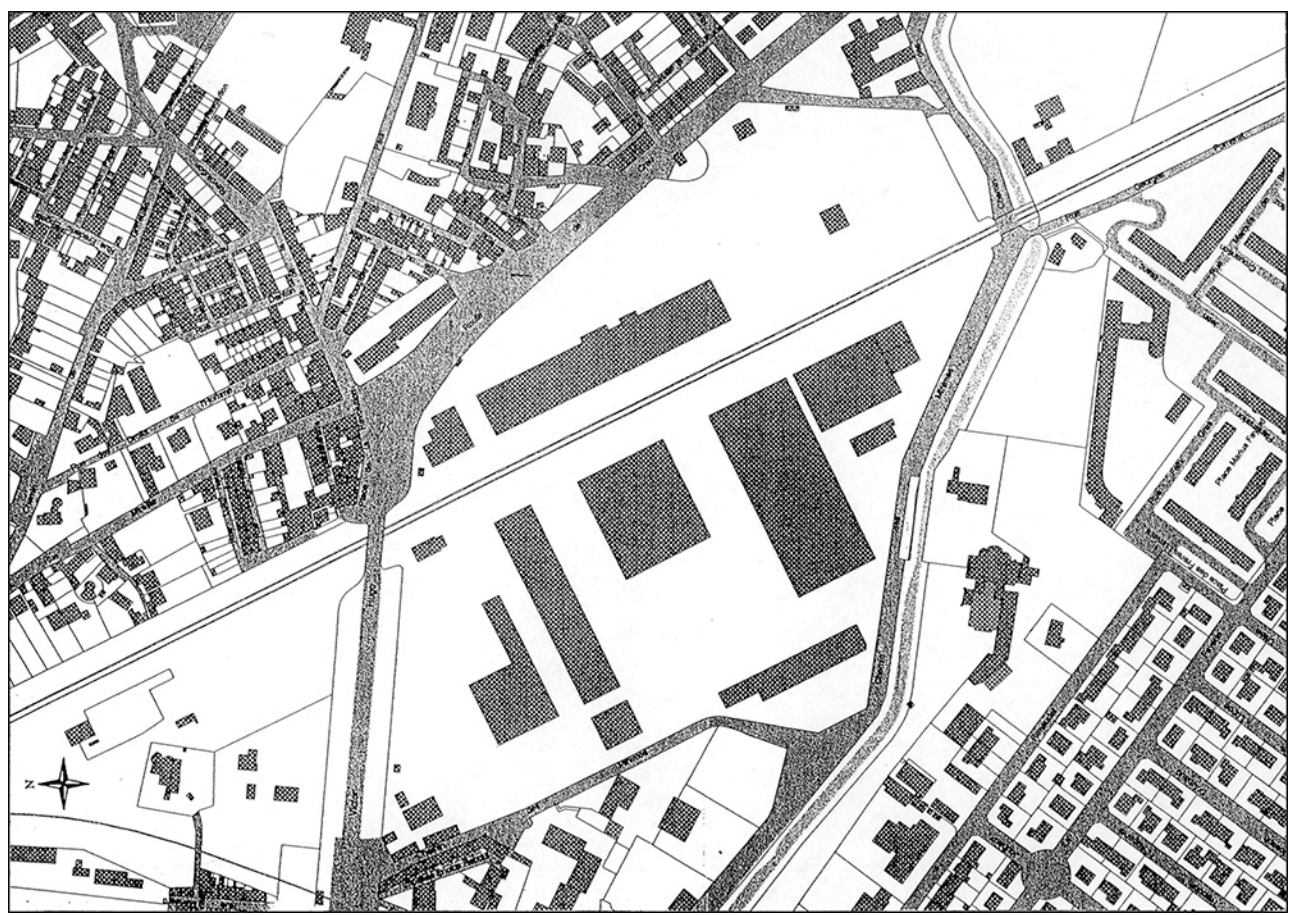

(c) Coralie Grégoire. 


\section{Le site des Ateliers d'Arles aujourd'hui, les vestiges matériels d'une évolution industrielle complexe}

\section{L'aboutissement de 136 ans d'activité industrielle ferroviaire}

Le site des Ateliers d'Arles, constructions et espaces libres, représente l'aboutissement de 136 ans d'activité et de mutations industrielles (fig. 3). Sur ces onze hectares se sont succédé de nombreux édifices en fonction de la conjoncture économique et de la politique de la compagnie gérant le réseau auquel appartiennent les Ateliers d'Arles. Quatre grandes périodes d'évolution de la gestion du bâti peuvent être distinguées.

Figure 3. Présentation synthétique des bâtiments successifs des Ateliers d'Arles.

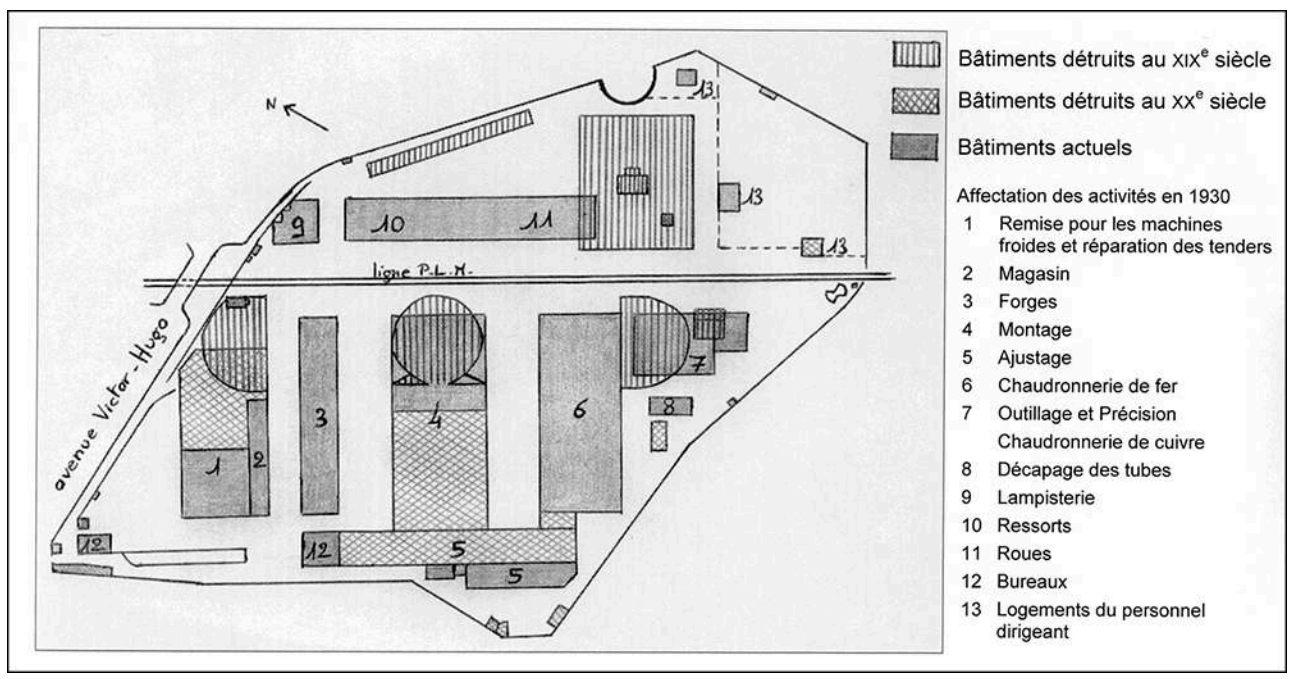

(c) C. Grégoire, 2001

Dès 1848 sont construites les premières halles destinées à la réparation et à la construction de locomotives à vapeur et de wagons, au stockage des matières premières et des pièces ainsi qu'une rotonde abritant un dépôt de matériel moteur.

En 1861-1862 se produit le premier remaniement, suite à l'essor du réseau ferré et aux successives concentrations de compagnies (1852: Compagnie Lyon-Marseille; 1857 : Compagnie Paris-Lyon-Méditerranée). Il correspond à un agrandissement du dépôt par l'ajout de deux demi-rotondes de part et d'autre de la rotonde d'origine.

Suite à la réunion de ses lignes, le PLM dispose de nombreux ateliers et dépôts répartis inégalement sur l'ensemble de son réseau. Il engage donc une politique de redistribution des forces sur ce réseau. Ainsi, se succèdent trois remaniements (1864-1865, 1872-1876 et 1884-1899) à la suite desquels les Ateliers d'Arles se consacrent aux locomotives, aux tenders et à la réparation des roues. L'atelier d'entretien des wagons est transféré à Nîmes en 1864-1865, le dépôt est transféré à Avignon en 1887 (Arles est rétrogradée au rang d'annexe-traction : une demi-rotonde est construite sur un terrain mitoyen, au nord des ateliers) et l'atelier des voitures est transféré à Oullins en 1889. Plusieurs bâtiments sont détruits, notamment l'ensemble du dépôt (rotonde et demi-rotondes), afin de permettre l'allongement des halles préexistantes ainsi que la construction de nouveaux édifices. 
6 Les Ateliers d'Arles conservent cet aspect jusqu'en 1953-1954, date de la démolition d'une large partie de deux bâtiments : l'ajustage et la remise des machines et tenders.

7 Dès 1913, malgré une apparence brillante, la situation financière des réseaux ferroviaires français, PLM inclus, est préoccupante: l'augmentation des charges de capital et de personnel, leur endettement croissant ont rendu la plupart d'entre eux déficitaires. En 1919, la Première Guerre mondiale relance la production des ateliers épargnés par le conflit. Cette année-là, les Ateliers d'Arles embauchent 670 personnes supplémentaires, ce qui porte les effectifs à 1769 ouvriers et employés. Mais les pertes occasionnées par le conflit sont considérables et le prix du charbon flambe. S'ajoutent à ces problèmes la toute récente concurrence des transports routiers et les mouvements sociaux de 1920 qui, aux Ateliers d'Arles, débouchent sur 470 licenciements. La crise économique des années 1930 ne fait qu'accentuer le phénomène. Le PLM tente de faire face en regroupant ses activités de réparation afin de maintenir un rendement convenable: les Ateliers d'Oullins, près de Lyon, sont choisis pour abriter le centre principal de réparation de matériel moteur. Entre 1931 et 1933, les Ateliers d'Arles perdent donc l'activité de réparation des locomotives au profit d'Oullins, d'autres ateliers et de l'industrie privée régionale. Les Ateliers d'Arles, en raison de l'accès difficile des locomotives modernes au montage et de la faiblesse des moyens de manutention, sont ceux qui réclameraient le plus de frais de modernisation dans l'hypothèse d'une poursuite de la réparation des locomotives. De 1934 à 1936, on assiste à une nouvelle vague de modernisation au sein des sections des roues, des ressorts, des forges, de l'ajustage et de la précision, mais dans l'optique de l'adaptation des ateliers à leurs nouvelles activités : la mécanique générale (pièces de rechange pour le matériel moteur, roulant ou fixe) et l'entretien (entretien de l'outillage, du matériel fixe et des bâtiments) pour le parc, le dépôt et le magasin.

8 Malgré un soubresaut d'activité après la Deuxième Guerre mondiale, la chute des effectifs est inexorable. L'activité du magasin se développe dans les années 1950 et les ateliers profitent de dotations de machines-outils modernes, mais cela ne change pas la donne, les activités se cantonnent à l'entretien et à la mécanique générale. On n'investit plus dans ces ateliers vétustes et inadaptés à la production de locomotives modernes Diesel ou électriques. L'ajustage et une partie de l'ancienne remise des locomotives et tenders sont même détruits, on l'a dit, en 1953-1954. Les Ateliers d'Arles sont jugés excédentaires dans le dispositif ferroviaire existant car leurs installations ne sont quasiment valables que pour des machines à vapeur et il n'est nul besoin de créer un autre grand atelier de réparation Diesel ou électrique. Ils sont donc uniquement utilisés pour décharger les grands ateliers de quelques travaux dans le but d'occuper la main-d'œuvre restante. Les effectifs s'amenuisent au fil des départs en retraite. Les Ateliers d'Arles ferment leurs portes le 31 décembre 1984, à un moment où l'industrie arlésienne est touchée par de nombreux autres sinistres.

\section{La complémentarité des archives}

9 Suite à leur fermeture en 1984, les Ateliers d'Arles ont été vidés de leurs équipements de production et de manutention. Aujourd'hui, seuls sont encore visibles quelques fosses de ponts roulants, dont une est encore équipée de son pont, quelques ponts roulants aériens et des chemins de roulement, ainsi que quelques vestiges de plaques tournantes. Pour prendre pleinement conscience de l'importance des équipements de 
production et de manutention, il est donc indispensable de faire appel aux données archivistiques.

Concernant les Ateliers d'Arles, il reste de nombreux documents élaborés à l'occasion des agrandissements ou des modifications de sections d'atelier, notamment à la fin du $\mathrm{XIX}^{\mathrm{e}}$ siècle et au début du XXe. Les plus intéressants présentent des plans, des coupes et des élévations de fondations relatifs à l'installation, au déplacement ou au retrait d'équipements de production et de manutention (machines-outils, grues, chemins de roulements, cuves, etc.) permettant de reconstituer, au moins partiellement, la mise en place et le fonctionnement des différents ateliers. En effet, des plans de groupes de machines et de moyens de manutention permettent d'établir l'organisation interne de ces sections d'atelier que sont par exemple les forges ou l'ajustage.

\section{Le bâtiment, une entité définissable et compréhensible au sein d'un ensemble : le site industriel}

\section{De puissants équipements de production}

11 Un peu plus de dix kilomètres de voie ferrée dessert les bâtiments et les aires de stockage. Cette densité impose la mise en place de dispositifs particuliers pour le tournage et l'orientation du matériel ferroviaire: cinq ponts roulants, un pont tournant, situé à l'extrémité sud des ateliers, qui oriente dans cinq directions différentes, ainsi que de nombreuses plaques tournantes, qui orientent ou font simplement office d'aiguillage. Plaques tournantes et ponts roulants sont tout aussi utilisés au sein des sections d'atelier pour les manutentions intérieures. S'y ajoutent des ponts roulants aériens pouvant supporter de très lourdes charges et circulant sur deux chemins de roulement aériens parallèles, des palans se déplaçant sur des chemins de roulement simples, des potences fixées aux piliers des bâtiments, et des grues fixes montées sur pivot ou des grues roulantes sur voie ferrée. Le type d'activité d'une section d'atelier conditionne sa dotation en moyens de manutention. De ce fait, le montage et la chaudronnerie de fer sont principalement équipés de puissants ponts roulants aériens, capables notamment de soulever les chaudières de locomotives. Dans l'atelier des forges, ce sont des grues montées sur pivot et des potences qui permettent le transport rapide sur de courtes distances des pièces chaudes entre les fours, les forges et les marteaux-pilons.

On retrouve aux Ateliers d'Arles les moyens de production caractéristiques des ateliers de construction mécanique de la première moitié $d u x^{e}$ siècle: des installations destinées au chauffage ou au réchauffage du métal (fours, forges), des machines pour le forgeage du métal, soit par percussion (marteaux-pilons), soit par pression ou par déformation (presses, machines à cintrer, à arrondir ou à plier), et des machines-outils utilisées notamment pour la finition des pièces. En général, une ou deux catégories d'équipements de production dominent au sein d'une section d'atelier. Ainsi, l'atelier des forges se compose plus particulièrement de fours, de forges et de pilons; la chaudronnerie de fer et la chaudronnerie de cuivre disposent davantage de machines pour le forgeage par pression, pour l'usinage de pièces parallélépipédiques, de fours et de quelques forges; quant à l'ajustage, il est exclusivement équipé de machines-outils 
pour le travail du métal à froid. L'atelier des roues est le plus diversifié en moyens de production : fours, forges, pilons, tours et machines-outils diverses. Situé sur la partie est des ateliers et séparé des autres sections de travail par les voies principales de la ligne du PLM, il doit être autonome dans ses activités. De grandes tables, des établis et des marbres complètent les installations mécaniques.

Sur ce site, on rencontre majoritairement deux types d'organisation des équipements de production au sein des sections d'atelier. L'organisation en travées est prédominante lorsqu'il s'agit de machines-outils, chaque alignement correspond à un des arbres de transmission de la machine motrice à vapeur. Cependant, l'organisation polycentrique est prépondérante dans l'atelier des forges : chaque four dessert un ou deux marteauxpilons et deux ou trois forges grâce à des potences et à des grues. Les deux systèmes peuvent cohabiter au sein d'une même section.

Du quatrième remaniement (1892-1899) à la veille de la Première Guerre mondiale, les Ateliers d'Arles ne cessent de moderniser et d'accroitre leurs équipements de production et de manutention. L'octroi de ces équipements peut résulter d'un programme de réaménagement d'une section d'atelier ou bien être ponctuel, il se limite alors à quelques unités. La modernisation des moyens de production est surtout due à l'utilisation et à la généralisation de nouvelles sources d'énergie. Durant le XIX siècle, l'essentiel de la force motrice des ateliers est produit par des machines motrices à vapeur, chaque section possède sa propre machine motrice qui dessert les moyens de production et de manutention grâce à des arbres de transmission aériens ou souterrains. Le gaz est employé parallèlement à la vapeur. Il est utilisé pour l'éclairage intérieur et extérieur, et pour le fonctionnement de certains équipements de production. La chaudronnerie de cuivre possède des fours, des forges et des chalumeaux à gaz, les forges disposent de quelques forges à gaz, et la lampisterie utilise une étuve à gaz, petit four destiné au séchage de la peinture ou du vernis.

Dès la fin du XIX ${ }^{e}$ siècle, de nouvelles sources d'énergie sont introduites puis se répandent. En 1897, l'éclairage électrique est installé à l'extérieur des bâtiments sur la partie est des ateliers et, en 1898, tout le site est ainsi équipé ; cependant, l'éclairage intérieur au gaz prédomine toujours. Le programme d'électrification générale des ateliers est lancé en 1908 et s'étend de 1910 à 1914. Plusieurs postes de transformation sont installés au sein des sections d'atelier et les machines motrices à vapeur sont retirées au fur et à mesure de leur mise en service. Lors de cette première phase d'électrification, à quelques exceptions près, les machines ne possèdent pas encore de moteur individuel. Un moteur électrique dessert un groupe défini de machines. Il faut attendre les années 1930 pour que les équipements de production soient dotés de moteurs individuels et qu'ainsi soient progressivement supprimées toutes les transmissions principales et intermédiaires.

Deux autres sources d'énergie se développent au $\mathrm{xx}^{\mathrm{e}}$ siècle, l'air comprimé et l'acétylène. Entre 1902 et 1911 sont installés les premiers compresseurs d'air à la chaudronnerie de fer. Au moyen de conduites et de prises d'air, ils desservent cette section et la précision voisine, qui disposera de ses propres compresseurs en 1931. L'air comprimé présente des avantages de puissance, de maniabilité et de sécurité, il est notamment utilisé pour actionner certains moyens de manutention comme les palans ; les ateliers possèdent aussi des marteaux pneumatiques. Il semblerait que l'usage de l'acétylène soit introduit simultanément, il est attesté en 1907. Cet hydrocarbure gazeux est fourni par des générateurs fixes appelés gazogènes et par des générateurs 
mobiles. Associé à de l'oxygène, comprimé dans des bouteilles, il est utilisé pour la soudure: l'acétylène brûle et l'oxygène l'active. La soudure oxyacétylénique ou autogène, rendant le travail de raccord de deux pièces bien plus simple et plus rapide, se substitue au rivetage. À partir de 1920, la soudure électrique ou soudure à l'arc remplace à son tour progressivement la soudure oxyacétylénique.

\section{L'organisation fonctionnelle des activités} l'entrée principale, donnant sur l'avenue Victor-Hugo, constituée d'un porche en pierre de taille encadré de deux édifices symétriques et surmonté d'une horloge. On distingue trois types d'occupation de l'espace au sein des Ateliers d'Arles. Tout d'abord, de vastes constructions, caractérisées par de grands volumes intérieurs, à vocation strictement industrielle. Se détachent ensuite de plus petits bâtiments, le plus souvent sur plusieurs niveaux, à usage administratif, social ou d'habitation ainsi que des zones non bâties.

En ce qui concerne les bâtiments à usage non industriel, deux secteurs leurs sont attribués, et ils le resteront au cours des décennies suivantes. À l'extrême Sud-Est, une large parcelle de terrain et trois habitations sont octroyées au personnel dirigeant des ateliers (ingénieur du Matériel, chef d'atelier, chef de dépôt...). Les constructions à vocation industrielle n'empièteront jamais sur cet espace. À l'extrême Nord-Ouest, à proximité du porche d'entrée, se concentrent les bâtiments à usage administratif (bureaux de l'arrondissement du Matériel, service médical puis service social, cantine). Les deux bâtiments flanquant ce même porche ont abrité dès le milieu du XIX ${ }^{e}$ siècle la loge des gardiens sur la gauche et le service médical sur la droite. En fait, cette zone est à la fois en contact direct avec la rue et isolée des activités productives. De plus sa déclivité (le seul endroit pentu des ateliers, situés en contrebas) est incompatible avec l'établissement de vastes bâtiments industriels.

Les espaces aujourd'hui non construits avaient chacun une fonction précise lorsque les ateliers étaient en activité. Les Ateliers d'Arles ayant été peu à peu mis à l'écart du circuit de production ferroviaire, certains bâtiments ont été détruits dans les années 1950 en raison de leur vétusté et une partie du montage a été rasée en 1985, suite à un incendie. Néanmoins, chaque parcelle de terrain non bâtie était utilisée: stockage, soutes à combustibles détruites après la fermeture des ateliers, parc à matrices, parc à roues; le tout disposé en fonction de l'aménagement des voies de passage des véhicules routiers et ferroviaires. Afin de limiter les déplacements des ouvriers et ainsi optimiser les rendements, les Ateliers d'Arles disposent d'aires et d'infrastructures spécialisées dans l'entreposage des matières premières, de l'outillage, des pièces de rechange, des pièces à réparer et des pièces finies. Des magasins intermédiaires et des cabinets d'outillage, installés au sein des principales sections, relaient le magasin principal. Celui-ci, installé dans un bâtiment sur deux niveaux, gère aussi des aires de stockage à ciel ouvert ou sous abri, quelquefois équipées de râteliers destinés au rangement de pièces précises (râteliers à tôles, à enveloppes de chaudières, à bandages). Signalons deux lieux d'entreposage plus particuliers: le parc à essieux de l'atelier des roues, formé d'un quadrillage de voies facilitant le rangement des essieux, et le parc à matrices de la section des forges, desservi par une grue roulante sur rails, le parc étant enserré entre les rails. 
20 Les parties est et ouest des ateliers sont séparées l'une de l'autre par la ligne principale du PLM. Avant la spécialisation dans la construction et la réparation des locomotives, tenders et essieux (1884-1899, quatrième remaniement), les différentes activités (dépôt, atelier des voitures, atelier des wagons, atelier des machines) se côtoient sur le site, mais occupent des zones distinctes. En 1856, la rotonde principale occupe le centre des ateliers et le montage lui est accolé afin de limiter les manœuvres. L'atelier des machines s'étend entre la rotonde et la clôture ouest, l'atelier des voitures occupe le Sud et l'atelier des wagons est isolé sur la partie est des ateliers (futur atelier des roues). Cette logique est conservée jusqu'à la spécialisation ; une exception, l'atelier des voitures qui sera pendant quelques années (1861-1865) à cheval sur les parties est et ouest. Étant donné la proximité des deux premiers remaniements (1861-1862 et 1864-1865), à l'issue desquels l'atelier des wagons est transféré vers Nîmes et Marseille, il semblerait que la compagnie ait scindé en deux phases les mutations permettant la concentration des activités de l'atelier des voitures du côté est des ateliers et le développement de l'atelier des machines à l'Ouest.

21 Les bâtiments du dépôt (la rotonde, les deux demi-rotondes et une remise) seront tous édifiés à proximité de la ligne principale de chemin de fer, les ateliers à proprement parler se trouvant en retrait. Cette logique de gestion de l'espace est assez courante. En effet, ce type d'infrastructure ferroviaire s'est établi lors des premières années de l'implantation du réseau ferré en France et dans le cadre de la politique de tronçonnement des lignes; cela oblige les compagnies à posséder sur de petites lignes toutes les activités indispensables à leur fonctionnement. Il est dans ce cas plus rentable de centraliser ces activités, notamment le dépôt et les ateliers.

La spécialisation progressive des Ateliers d'Arles dans la construction et la réparation des machines à vapeur (achevée par le quatrième remaniement de 1884-1899) a permis d'occuper l'espace laissé vacant par le départ des autres activités, sans avoir à s'agrandir au-delà de l'enceinte d'origine. Situés dans une vaste cuvette creusée à cet effet lors de la construction de la ligne Avignon-Marseille, ils sont composés de deux parties distinctes séparées par cette même ligne de chemin de fer. La partie est est consacrée à la réparation des roues du matériel moteur et roulant. Étant donné son isolement géographique par rapport à la partie ouest, elle dispose, on l'a dit, d'un équipement de production complet lui assurant une certaine autonomie (forges, tours, cuves à lessiver, fours, diverses machines-outils, un magasin propre). Sur la partie ouest se concentrent les activités relatives aux locomotives et aux tenders, ainsi que le magasin général. Les différentes sections d'atelier forment un ensemble ordonnancé autour d'un cœur, le montage, qui leur distribue les pièces à réparer et décide de la fabrication de nouvelles pièces. Le montage est encadré par les forges d'une part et par la chaudronnerie d'autre part, ce qui permet de ne pas manœuvrer sur de trop longues distances les plus lourdes pièces telles que les chaudières de locomotives. Perpendiculairement à ces trois bâtiments et contigu au montage et à la chaudronnerie se place l'ajustage par lequel passent de nombreuses pièces afin d'être usinées avant de retourner au montage pour être ré-assemblées.

23 À ce moment-là, les ateliers arrivent à leur point d'extension maximum. Dans les années suivantes, les remaniements d'activités ne se manifestent plus que par des réorganisations au sein des bâtiments existants.

Les ateliers de réparation ferroviaire d'Arles ne sont plus présents que par leur aspect architectural, la plupart des équipements de production ayant logiquement été 
transférés lors de la fermeture vers d'autres ateliers en activité. Ce site, plus aisément abordable et bien plus visible que les archives, est l'expression matérielle de près d'un siècle et demi de travail humain. Les bâtiments, les espaces libres, l'agencement des premiers par rapport aux seconds forment un tout indissociable au sein duquel chaque parcelle, qui est le résultat de dizaines d'années d'activité industrielle, acquiert ainsi sa signification. Dans le cadre d'une revalorisation du site des Ateliers d'Arles et à défaut d'une protection au titre des monuments historiques, il serait plus que préférable de conserver l'ensemble du site afin de préserver son unité et sa cohérence.

\section{BIBLIOGRAPHIE}

\section{Orientation bibliographique}

- Coralie Grégoire, Les Ateliers d'Arles de 1848 à 1984, 136 ans d'activité industrielle au service de la cause ferroviaire, mémoire de maîtrise d'histoire contemporaine, sous la direction de Philippe Mioche et Claude Massu, Université de Provence, 2001, étude réalisée avec l'aide scientifique et financière de l'Association pour l'histoire des chemins de fer en France (AHICF).

- Christian Chevandier, «L'abandon de la traction vapeur : les mutations professionnelles dans les ateliers du Matériel aux lendemains de la Seconde Guerre mondiale », in « Hommes et machines, techniques et métiers du rail », Actes de la journées scientifique du 2 juin 1992, Revue d'histoire des chemins de fer, 8 (automne 1992).

- Auguste Perdonnet et Camille Polonceau, Portefeuille de l'ingénieur des chemins de fer, Paris, Librairie scientifique industrielle de L. Mathias, 1843-1846.

- SNCF Région Méditerranée, Les Ateliers d'Arles, historique, 1954.

\section{RÉSUMÉS}

Aujourd'hui, les ateliers de réparation ferroviaire d'Arles ne sont plus présents que par leur aspect architectural, la plupart des équipements de production ayant logiquement été transférés lors de la fermeture du site vers d'autres ateliers en activité. Laissé libre, l'agencement des bâtiments et des espaces forme un tout indissociable au sein duquel chaque parcelle, résultat de dizaines d'années d'activité industrielle, est porteuse de signification.

À partir du site mais aussi d'un corpus archivistique, l'article propose dans un premier temps un retour historique sur le développement et l'activité des ateliers d'Arles depuis l'ouverture en 1848. Les profonds remaniements opérés entre les années 1860 et 1880 traduisent la spécialisation progressive du site dans les locomotives, les tenders et la fabrication de roues avant le recentrement, dans les années 1930, sur la mécanique générale et l'entretien. Les difficultés économiques et structurelles croissantes rencontrées par le PLM avant 1914 et dans l'entre-deux-guerres entraînèrent un déclin inexorable du site d'Arles, dont la modernisation et les mutations continues (mais toujours partielles, au bénéfice des après-guerres notamment) ne purent être réellement décisives. 
Dans un second temps, l'auteur traite du patrimoine bâti qui occupe ou occupa un site en constante évolution. L'article en éclaire les ressorts techniques et fonctionnels par la succession des équipements, outils et machines qui permet de comprendre l'organisation spatiale avant tout fonctionnelle de l'atelier. Excédentaire et dépassé dès la reconstruction, le site est devenu depuis sa fermeture en 1984 un objet patrimonial et pédagogique remarquable pour l'histoire ferroviaire et, au-delà, l'histoire du travail.

Nowadays, the rail repair workshops of Arles are no more than an architectural appearance. Most of the production equipments have logically been transferred to other workshops in activity during the closure of the site. Left free, the layout of buildings and spaces forms an inseparable whole in which each place, result of decades of industrial activity, is meaningfull.

From the site reality and also an archival corpus, the author proposes first a historical overview on the development and activity of the workshops of Arles since the opening in 1848. The major changes between the 1860's and 1880's reflect the progressive specialization of the site in the locomotives, the tenders and the manufacture of wheels before the refocusing, in the 1930's, on general mechanics and maintenance. The growing economic and structural difficulties encountered by the PLM before 1914 and in the interwar period led to an inexorable decline of Arles site, in spite of undecisive modernization and continued mutations (still partial, in post-war period essentialy).

In a second time, the article deals with built heritage which occupies or occupied a site in constant evolution. Through the succession of equipment, tools and machines, the author enlightens the technical and functional history of the site to understand the spatial organization (essentialy functional) of the workshops. Already surplus and obsolete at the reconstruction, the site has become, since its closure in 1984, an heritage place and a remarkable educational tool for the comprehension of the railway history and, beyond, the labour history.

\section{INDEX}

Keywords : architecture, city, collective memory, industrial heritage, historical monument, Provence, railway infrastructure, town and country planning, workshop

Mots-clés : aménagement, architecture, atelier, infrastructure ferroviaire, mémoire collective, monument historique, patrimoine industriel, Provence, ville

\section{AUTEUR}

\section{CORALIE GRÉGOIRE}

Maître en histoire, université de Provence, diplomée du DESS « Gestion et valorisation $\mathrm{du}$ patrimoine industriel », université de Bourgogne 